\title{
Stochastic Modelling of Electrical Tree Progression in Modern High Voltage Power Cables
}

\author{
M. Hariharan \\ Indira Gandhi Centre for \\ Atomic Research Kalpakkam, 603102, \\ Tamilnadu, India
}

\author{
Sharanya Srinivas \\ Department of \\ Electrical Engineering \\ Arizona State University \\ (ASU) Tempe, Arizona, USA
}

\begin{abstract}
A complete theoretical model of pre - breakdown phenomenon in indoor polymeric insulators is demonstrated. It has been observed that occurrence of breakdown in solid dielectrics is mainly due to the progression of electrical treeing which is eventually generated due to localized field enhancement and partial discharge activity. A methodical analysis of tree propagation in XLPE cables is performed by adopting two dimensional stochastic simulations. The major advantage behind this model is that it inherently exhibits parallel processing by considerably reducing the computation time. It has been observed that when the probability of the electric field at the needle plane exceeds the critical electric field the material degrades due to the inception of fine filamentary tree channels. Using standard needle plane geometries, two main types of tree structures, bush type and branch type trees were simulated. Fractal dimensions of the simulated electrical trees were being accurately computed. Failure analysis of electrical trees is investigated by adopting weibull - distribution studies.
\end{abstract}

\section{General Terms}

Power Cables, Cable Insulation, Failure Analysis

\section{Keywords}

Partial Discharge (PD), Electrical Breakdown, XLPE, Correlation Constant, Stochastic Model, Weibull Distribution

\section{INTRODUCTION}

Electrical treeing is one of the most important breakdown mechanisms which occur due to initiation of failure in insulating materials. Electrical trees are initiated due to high electric stress enhancing factors like the nature of voids, material properties and respective needle plane geometries. The growth of electrical tree is of progressive type and damage occurs locally. To understand the behaviour of the tree growth, it is possible to utilize the characteristics of Stochastic Modelling techniques. This Stochastic Model is a computational process consuming ample execution time. The salient feature is that the finite element algorithm enables parallel processing inherently. Various statistical models were being developed to understand the level of tree propagation in insulating materials. It has become evident that tree propagation is affected mainly due to partial discharge activity. In this present work, a stochastic model has been developed to investigate the level of partial discharge within the tree channels. During partial discharge charge is being transferred to the walls of the tree channels. Considerable efforts were made to simulate the effects of partial discharge on tree propagation.

\section{MODELLING ASPECTS OF ELECTRICAL TREES}

Electrical trees are stochastic structures, they could be bushy or a single branch type in nature initiating from the needle plane. Trees consists of gas filled channels which normally gets originated from existing voids, cavities and other material defects, which continues to propagate inside the material by variable means, one of which is due to partial discharge at various points inside the grid which is due to high electric stress. Trees generally initiate from a high - field region and start to propagate through the dielectric by forming fine filamentary tube channels due to rapid degradation of the insulating material. Experimental analysis from world over researchers has shown that electrical treeing is intrinsically linked to partial discharge activity within the tree tubules. During the simulation of tree structures various considerations were taken into account that the tree propagates inside a dielectric when the dielectric strength is lesser than the applied electric stress. The so called NPW model (named after its inventors Niemeyer, Pietronero and Wiesmann) allows ease of simulation on a digital computer using finite element approach. The original NPW model being enhanced by Barclay et al. has undergone considerable changes to obtain the tree structures that match exactly with the actual Lichtenberg (2D) figures. These modifications incorporate a intrinsic breakdown phenomena for ionized particles inside a tree channel. The general simulation procedure of electrical treeing is an iterative process where electrical stresses are repetitively calculated at points of interest in a material. The medium of the material is divided into smaller grids or neighbourhood mesh elements and the material properties were being calculated. In particular the solution of Laplace equation is taken into consideration for the electric field when there is no consideration for space charges whilst the solution of Poissons equation is taken into account where the space charges are permitted inside the medium. To account for the probabilistic observation for the growth of electrical trees, a stochastic model is used to determine the dielectric strength throughout the medium as a function of its material property [2]-[5]. Figure 1 summarizes techniques and modelling considerations with respect to electrical treeing. 


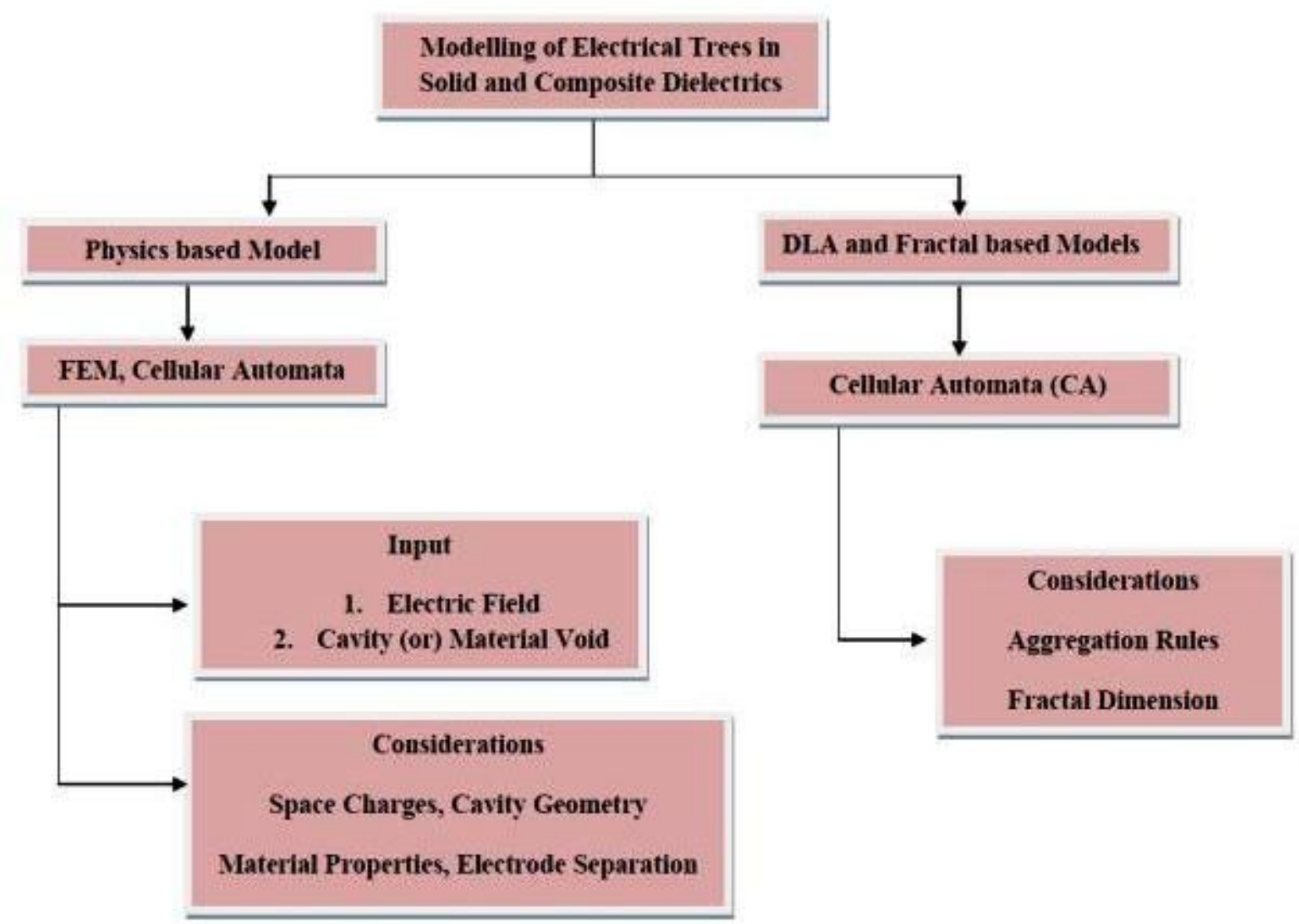

Fig 1: Summary of Electrical Treeing simulation techniques considerations and constraints

\section{SIMULATION STUDIES}

Consider an nxn grid assuming two electrodes being present at the top and bottom which resembles like the capacitance plates. Where the top electrode being the HV electrode and the bottom being the ground electrode.

- Assume that the HV electrode posses the highest potential of $1 \mathrm{~V}$ and the ground electrode posses potential of $0 \mathrm{~V}$. The space between the respective electrodes is considered as dielectric.

- In order to simulate the electrical tree, boundary conditions need to be assumed for the nxn grid points.

- Here Dirichlet boundary condition is taken into consideration to determine the boundary value for the grid points.

$$
\frac{\partial u}{\partial x}+\frac{\partial v}{\partial y}=0
$$

- A uniform gap of approximately ' $n$ ' lattice points is considered between the electrodes.

- An assumption is made that a needle electrode is present at the midpoint of the nxn grid with highest potential of 1V. At breakdown condition the tree tends to propagate from the needle plane to the ground electrode.

- Near the highest potential point along tip of the needle plane assume 3 points at 3 different directions and compute the electric field at these three points using solution of Poissons and Laplace equation.

$$
\begin{gathered}
\frac{\partial N e}{\partial t}=\frac{\partial(N e W e)}{\partial x}+\frac{\partial}{\partial x}\left(\frac{D \partial N e}{\partial x}\right) \\
\operatorname{grad} V=\frac{\partial}{\partial x}\left(\frac{\partial v}{\partial x}\right)+\frac{\partial}{\partial y}\left(\frac{\partial v}{\partial y}\right)=-\frac{\rho}{\varepsilon}
\end{gathered}
$$

- The site and direction of the tree progression from the needle plane geometry is chosen at random, but the probability of any site and direction being chosen is proportional to the function of electric field at the site in that direction. It is generally calculated by using the power law equation.

$$
\mathrm{P}=\mathrm{E}^{\eta}
$$

- Assumption is made that the electric field at these three points is localized.

- Then the electric field obtained at these three points was normalized using mathematical calculations. At these three points different normalized electric field exists, such that all the three points were added to obtain the sum of normalization of the electric fields to be equal to 1.

- After normalization random numbers are being generated across the nxn grid for the electric field that exists between $\mathrm{HV}$ electrode and the ground electrode and it is assumed as critical electric field.

- The neighbourhood points in the grid that are greater than the value of the random number generated at time step ' $t$ ' were assumed to be the sites for propagation of the tree. 
- A very important assumption made here is that the potential of the connecting links are the same as that of the top electrodes, which means that there is no potential drop along the discharge paths.

- ' $n$ ' number of lines with a specified length ' $L$ ' were drawn connecting these points and subsequently the midpoints of each line were determined.

- An assumption is made that the potential of highest electric field is 1 and then the three random numbers of the electric fields being obtained is compared with other neighbouring points to determine at which point the probability of the electrical field is highest.

- Then it is assumed that initially within these three points the tree starts to propagate.

- $\quad$ Again using the generated random numbers three more points were added to the grid to determine the other points where the probability of the electric field is highest. This entire process is iterative and is achieved by using Monte - Carlo Simulation.

- The iterative formula for electrical tree propagation using stochastic modelling is mentioned below

$C_{n+1}(i, j)=F\left(C_{n}(i-1, j-1), C_{n}(i-1, j), C_{n}(i-1\right.$, $j+1), C_{n}(i, j-1), C_{n}(i, j+1), C_{n}(i+1, j-1), C_{n}$ $\left.(i+1, j), C_{n}(i+1, j+1)\right)$

Where $C_{n}(i, j)$ and $C_{n+1}(i, j)$ are the states of the $(i, j)$ cell at time steps $t$ and $t+1$ respectively. By using the states of the neighbours, all the cells on the lattice were updated simultaneously.

\section{DIMENSIONAL ANALYSIS OF ELECTRICAL TREES}

The fractal analysis of electrical trees is being analyzed by modern digital image processing methods. The analysis is done by one of the subordinate methods of image processing technically called as image construction and image restoration models. Fractal dimension is purely a statistical measure of the extent of area that the tree tends to propagate.

In this approach Sandbox method is adopted to analyze the fractal dimension using statistical image processing techniques. This method is purely spatial in nature, operating on the completed tree pattern.

The dimension is obtained by adding the function

$\mathrm{M}=\mathrm{M}_{0}(\mathrm{t}) \mathrm{R}_{\mathrm{t}} \mathrm{D}_{\mathrm{f}}$

to the data, where $\mathrm{M}_{0}$ and $\mathrm{R}_{\mathrm{t}}$ are the parameters relating to mass and radius respectively. When the results are presented on a $\log$ - log plot, the function appears to be a straight line completely fitted to the grid points with gradient $\mathbf{D}_{\mathbf{f}}$.

The parameter $\mathbf{D}_{\mathbf{f}}$ computed is the fractional dimension. The fractal behaviour holds only over a certain range of scales for any given tree. From the observations it was clearly noted that breakdown has occurred at the two extremes, where the lower represents the bond length and the upper being the gross size of the tree. It is valid upto a limited range of limits of fractal scales.

The dimension estimates computed is being modified at every extreme when the power law is fitted, such that all the estimates lie within the fractal range. The dimension estimates being obtained from the fractal analysis of the simulated electrical trees using sandbox method has been tabulated below. From the table we can observe that for both internal and critical electric fields the values obtained varies approximately by a small value $\Delta$.

Table 1: Fractal Dimension estimates for each value of $\eta$ with $\mathbf{E}_{\text {int }}=\mathbf{E}_{\text {Crit }}=0$

\begin{tabular}{|c|c|}
\hline $\mathbf{\eta}$ & Sandbox Method \\
\hline 0.5 & $1.9241+/-0.1071$ \\
\hline 1.0 & $1.821+/-0.037$ \\
\hline
\end{tabular}

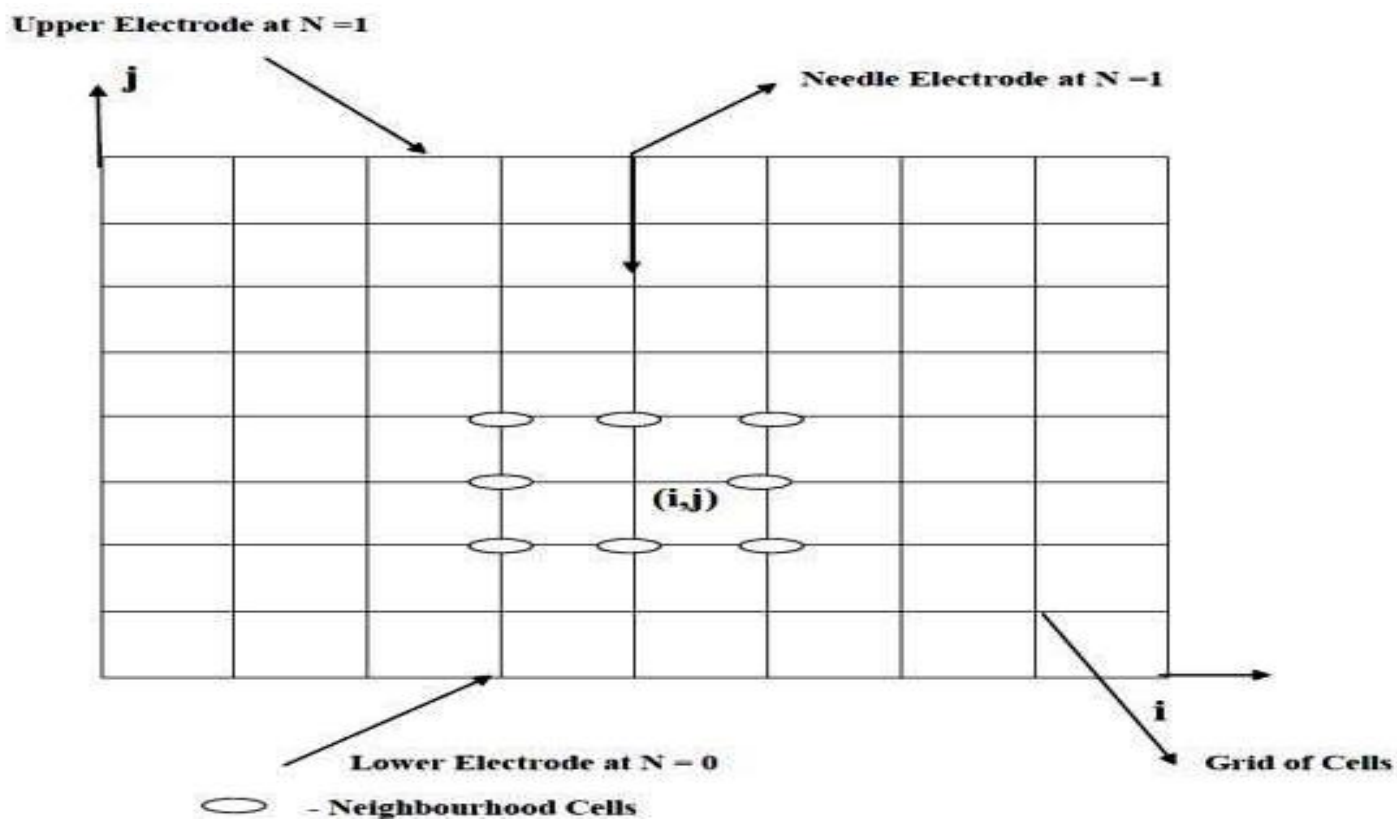

Fig 2: Neighbourhood grid points of the cell $(i, j)$ 


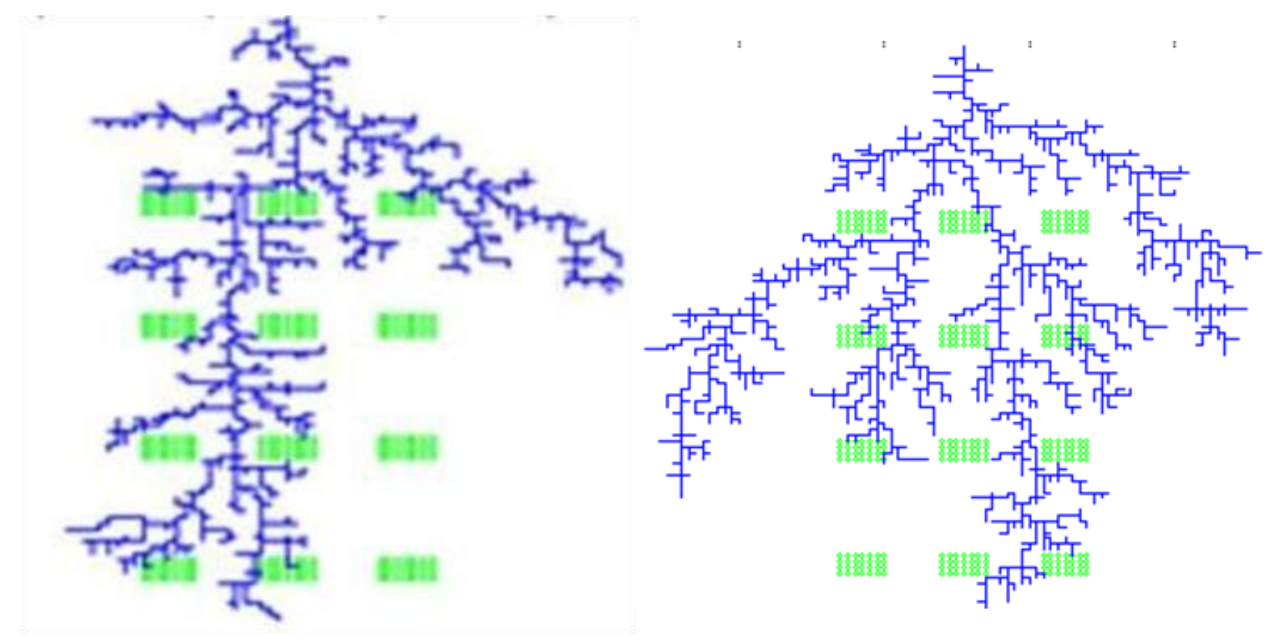

Fig 3: Simulated Electrical Tree Structure

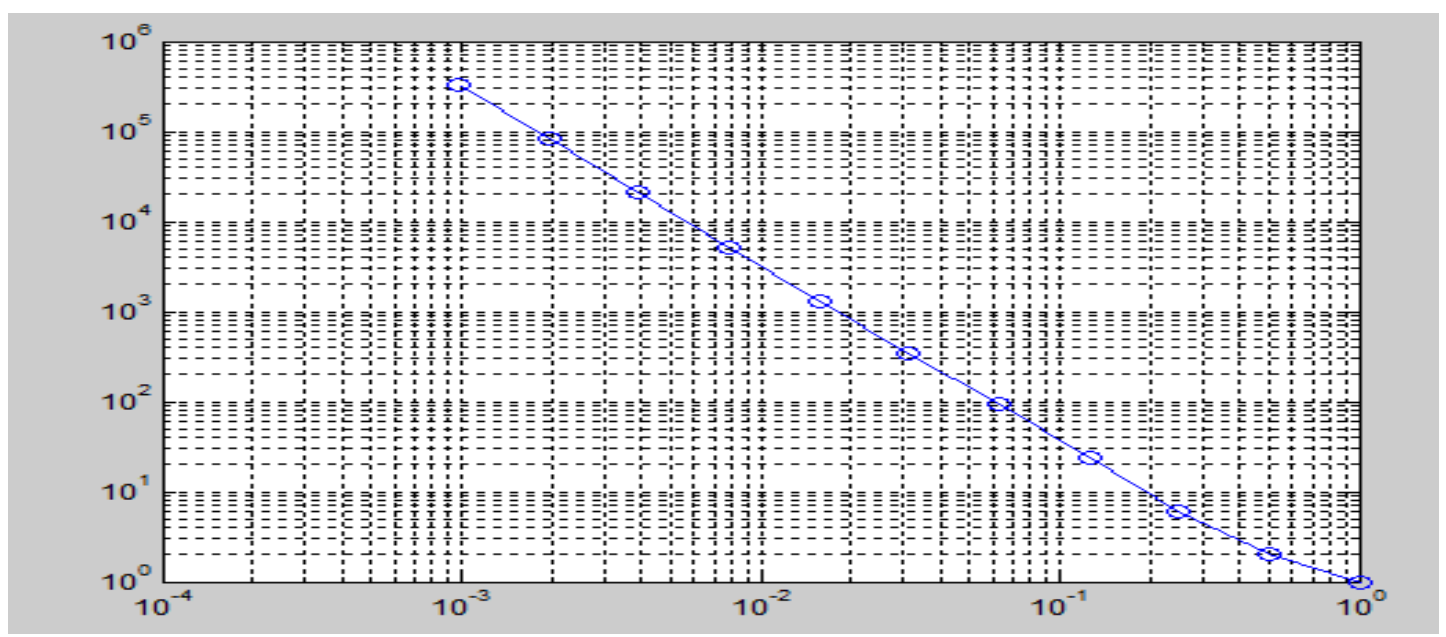

Fig 4: A Typical $(\ln N(\varepsilon) / \ln \varepsilon)$ plot for analysis of dimension estimates of an electrical tree

\section{CONCLUSIONS}

The numerical simulation of breakdown phenomena in insulating material using stochastic modelling is presented in this paper. In the present work the simulations have been limited to two dimensions. Various tree structures can be simulated from a defect site namely bush type, branch type and fibrillar type trees depending on the level of applied voltage. The presence of voids inside the material initiates damage due to partial discharge and overall it affects the level of tree progression. It is observed that bush - branch trees are likely to get developed due to partial discharge activity inside the tree channels. Fractal dimension estimates of the simulated electrical trees were obtained using the sandbox analysis. Weibull distribution studies clearly indicate that the life of the insulating material considerably gets reduced due to high electric stress. Essential investigations were required to establish the correlation between the accumulation of space charges and partial discharge activity. As a scope of future work this simulation can be extended to investigate the mechanism of electrical treeing and breakdown in nanodielectrics.

\section{ACKNOWLEDGEMENTS}

We are extremely grateful to Prof. Dr. R.Sarathi, Department of Electrical Engineering, IIT- Madras, for extending his kind support to carry out the present study.
One of the authors (M.Hariharan) wishes to thank Mr. L.Subramanyam, Scientific Officer, Head Electrical Works Section (EWS), Department of Atomic Energy (DAE), Indira Gandhi Centre for Atomic Research, Kalpakkam for his consistent support and guidance throughout this work.

\section{REFERENCES}

[1] A.L.Barclay, P.J.Sweeney, L.A.Dissado and G.C.Stevens, "Stochastic Modeling of Electric Treeing: Fractal and Statistical Characteristics" Journal of Physics D: Applied Physics, V23, pp.1536-1545, 1990.

[2] L.A.Dissado, S.J.Dodd, J.V.Champion, P.I.Williams and J.M.Alison, "Propagation of Electrical Tree structures in Solid Polymeric Insulation", IEEE Trans. DEIS, V4, pp 259-279, 1997.

[3] J.V.Champion and S.J.Dodd, "An approach to the Modeling of Partial Discharges in Electrical Trees", J.Phys.D: Applied Physics. Vol 31, pp.2305-2314, 1998.

[4] M. G. Danikas, I. Karafyllidis, A. Thanailidis, A. M. Bruning, "Simulation of electrical tree growth in solid dielectrics containing voids of arbitrary shape", Modelling and Simulation in Materials Science and Engineering, vol. 4, no. 6, 1996.

[5] M.D.Noskov, A.S.Malinovski, M.Sack, and A.J.Schwab, "Self-Consistent Modeling of Electrical 
Tree Propagation and PD Activity",IEEE Trans. On DEIS, Vol 7, No.6, pp.725-733, 2000.

[6] H. J. Wiesmann and H. R. Zeller, "A fractal model of dielectric breakdown and pre-breakdown in solid dielectrics", J. Appl. Phys., v60, pp. 1770-1773, 1986.

[7] K. Wu, Y. Suzuoki, T. Muzutani and H. Xie, "Model for partial discharges associated with treeing breakdown: I. PDs in tree channels", J. Phys. D: Appl. Phys., v34, pp. 1389-1398, 2001.

[8] T. Farr, R. Vogelsang, K. Frohlich, "A new deterministic model for tree growth in polymers with barriers," Electrical Insulation and Dielectric Phenomena, 2001 Annual Report. Conference on, vol.,no., pp.673-676, 2001.

[9] K. E. Seralathan, A. Mahajan, N. Gupta, "Modeling of electric tree progression due to space charge modified fields", Journal of Physics D: Applied Physics, vol. 41, 2008 .

[10] Seralathan K E and Gupta N, 2006: "Stochastic modelling of electric tree progression due to partial discharge activity "Int. Conf. on Properties and Applications of Dielectric Materials (Bali, Indonesia) vol 1 pp 119-22.

[11] Michael G. Danikas, Toshikatsu Tanaka:" Nanocomposites - A Review of Electrical Treeing and Breakdown": DEIS Feature Article: July/August 2009 Vol. 25, No. 4

[12] D. Antony Louis Piriyakumar, Paul Levi, R. Jayaganthan, R. Sarathi, "A Parallel Processing Technique for Electrical Tree Growth in Solid Insulating Materials Using Cellular Automata", Int. Conf. on Parallel Computing in Electrical Engineering, IEEE, pp 228-231, 2000 